\title{
Knowledge Management Applied to E-government Services: The Use of an Ontology
}

\author{
John Fraser ${ }^{1}$, Nick Adams ${ }^{1}$, Ann Macintosh ${ }^{1}$, Andy McKay-Hubbard ${ }^{1}$, \\ Tomás Pariente $\mathrm{Lobo}^{2}$, Pablo Fernandez $\mathrm{Pardo}^{2}$, Rafael Cañadas Martínez ${ }^{2}$, and \\ Jesús Sobrado Vallecillo ${ }^{2}$ \\ ${ }^{1}$ International Teledemocracy Centre, Napier University, 10 Colinton Road, Edinburgh, \\ EH10 5DT, Scotland \\ \{j.fraser, n.adams, a.macintosh, a.mckay- \\ hubbard\} @napier.ac.uk \\ ${ }^{2}$ Indra A.S., Avda. de Bruselas 35, 28108 - Arroyo de la Vega - Alcobendas, Madrid, \\ España \\ \{tpariente, pfpardo, rcanadas, jmsobrado\}@indra.es
}

\begin{abstract}
This paper is about the development and use of an ontology of egovernment services. We identify the knowledge required to deliver egovernment transaction services. Based on the SmartGov project, we describe the use of a domain map to assist in knowledge management and motivate the use of an ontology as a domain map. We describe the development of the egovernment service ontology and give a few examples of its definitions. We explain why the SmartGov project has adopted taxonomies, derived from the ontology, as its domain map. We highlight issues in ontology development and maintenance.
\end{abstract}

\section{Introduction}

Delivery of complete e-government services requires public authorities to be able to:

- publish information that customers (citizens, private sector and other public authorities) can use

- gather information through usable forms

- react online to specific requests from customers

- manage the online exchange of items of high-value

- integrate services as much as possible

A large amount and wide range of knowledge is required to achieve this. The knowledge is applied by managers, service designers and those who operate and support the services, including information technology (IT) specialists.

This paper examines how such knowledge can be managed for the benefit of all the roles in the previous paragraph and, ultimately, customers. It is based on work done during the SmartGov project, the main deliverable of which is to be a knowledgebased software platform for design and delivery of e-government services.

First we examine the knowledge requirements in more detail and describe SmartGov. In particular we explain how a domain map and knowledge units are used

The original version of this chapter was revised: The copyright line was incorrect. This has been corrected. The Erratum to this chapter is available at DOI: 10.1007/978-3-540-44836-5_33

M.A. Wimmer (Ed.): KMGov 2003, LNAI 2645, pp. 116-126, 2003.

(C) Springer-Verlag Berlin Heidelberg 2003 
in SmartGov. We then motivate the use of an ontology as a domain map and explain how we derived the SmartGov e-government service ontology.

We acknowledge the difficulty in using the full ontology as a domain map and describe the use of taxonomies as an alternative. Finally we discuss our experiences, with reference to: the use of other ontologies; involving users; and maintaining ontologies.

\section{Knowledge Management in E-government Services}

\subsection{The Knowledge Required}

Delivering a service, whether online or offline, requires a great deal of diverse knowledge: about customers, their needs, their behaviour, applicable legislation, available resources, working practices, successes and failures, other services, etc.

Stages of e-government services are often distinguished. See, for example, [1-4]. The following examples of knowledge refer to some commonly identified stages:

\section{publishing - one-way communication}

Here, knowledge is required about how to present information clearly online, how to manage its publication and how customers are likely to use the information. Knowledge may be required about the design, completion and processing of forms, any of which may be supported by software. For example, if customers are offered a template that they can complete using software on their own computers, then knowledge about how to guide and constrain the completion process is required.

\section{interacting - two-way communication}

Here, knowledge is required of how to react "electronically" to requests from customers. This may include knowledge of how customers search for information and like to receive it; how to make bookings on behalf of the customer; or how to accept and maintain customer information. Issues of security may become important.

\section{transaction - exchange of resources of nominally higher value than information}

The distinguishing feature of this stage is usually the secure online exchange of items other than information, for example taxes, registration fees and licences. The knowledge required is concerned with the security and efficiency of the transactions. Efficiency is often achieved by smoothly interfacing the online system with backoffice processing systems. People become more aware of issues such as trust, and the detail of the processes in which they are engaging.

\section{integration - all aspects}

"Integrate" is usually used here in the sense of integrating the provision of many, or all, of the offered services. This should lead to the blurring of distinctions in the eyes of the customer, e.g. which department provides a particular service or holds particular data, or where one "service" ends and another begins. Here knowledge is required of how to streamline and coordinate the design and delivery of services that already have all the required attributes from the previous stages. 


\subsection{An Example: The SmartGov Project}

SmartGov is a project that aims to take some of the effort out of managing the knowledge. It is particularly relevant to the transaction and integration stages.

SmartGov will produce a knowledge-based core repository for government transaction services that is usable by public authority staff. It will store, in knowledge units, the kinds of knowledge identified above and make them available to those staff as services are being developed, deployed and maintained.

A knowledge unit is anything worth storing that may help things to be done better in the future: help, best practice guidelines, examples, stories, lessons learned, troubleshooting advice, or training material. They can be of any size. Here are some short examples:

A sensible choice of questions on this form can make it easy for people to fill in. I would suggest these: "What is your country of origin?", "Is English your first language?"

People are often reluctant to fill in these details. This is a legacy from the days of the Poll Tax.

Michael Marra in the Urban Sustainability (US) group has written a good guidebook for this. Look on the intranet under Local Guidelines.

In SmartGov, we are accommodating unstructured knowledge units. We ask contributors merely to state which type of knowledge they are adding - guideline, lesson learned etc. - without analyzing the content of the knowledge unit any further. An area worth exploring in future is how to help users to structure their contributions for more fruitful sharing. In [5], for example, the use of terms such as originating action, conditions, contribution and result are proposed to describe lessons learned.

In SmartGov, the structure of the whole knowledge base is designed to reflect the domain of transaction services, as follows.

Knowledge units can be associated directly with the various components of electronic transaction services:

- transaction service element, i.e. a placeholder for data

- group of transaction service elements, e.g. the set of placeholders for company VAT registration data

- form, within which transaction service elements and groups are placed

- the whole transaction service

Knowledge units can also be associated indirectly with the transaction service components listed above, through a structure that defines the basic concepts and relationships of a domain. We refer to this structure as the domain map. Knowledge units can be associated with concepts and relationships in the domain map; the transaction service components can also be associated with concepts and relationships in the domain map. See Fig. 1 


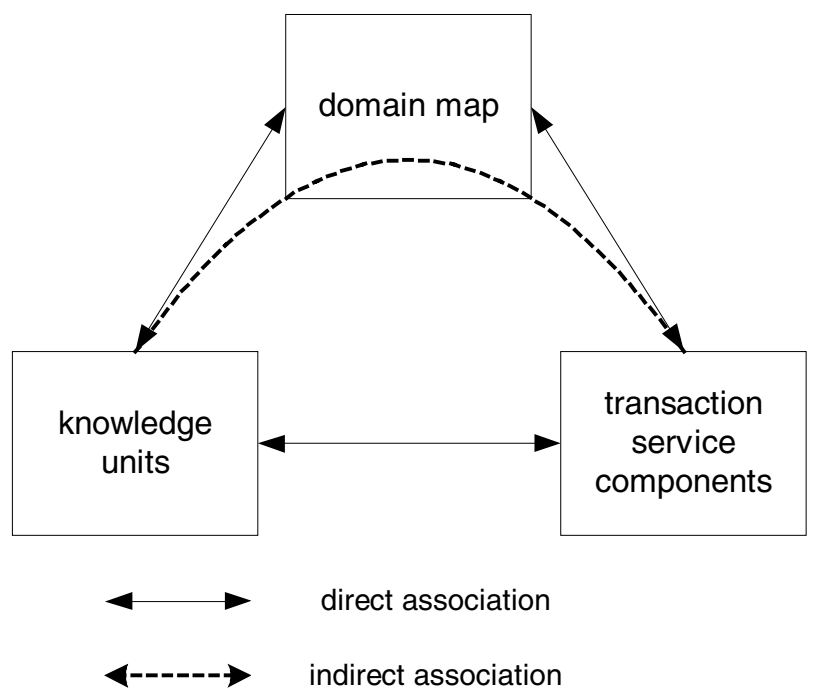

Fig. 1. Knowledge unit associations

The domain map, therefore, defines a conceptual framework with which to describe the components of a service and the knowledge associated with all aspects of the service from design to maintenance.

In SmartGov, the delivered platform - ready for use by public authority staff will contain a defined domain map and existing knowledge units, with appropriate associations between them. There will also be a small library of template transaction service components, again with appropriate links.

Design of a new service entails the user making copies of service components and creating new ones. As this happens, the user is given access to knowledge units that are associated either directly or indirectly with the template components.

It is anticipated that new knowledge units will be added frequently by users, who can decide on associations between knowledge units and the other parts. The domain map may also change over time, but much more slowly.

As services get developed, therefore, the example knowledge units listed above might be associated directly with domain map elements such as:

knowledge unit

A sensible choice of questions...

People are often reluctant to...

Michael Marra in the ...

\section{domain map elements}

ethnic origin, registration

tax, claim, benefit, budget

city, environmental provision

and with transaction service components such as:

knowledge unit

A sensible choice of questions...

People are often reluctant to...

Michael Marra in the ... transaction service components ethnic origin group, library registration form home address group, council tax service environmental assessment service

Then, whenever a user of the platform is designing a registration form, the "sensible choice of questions" knowledge unit is available to the user if desired. 


\section{Ontology as a Domain Map}

One of our activities in the SmartGov project has been to build an ontology for egovernment services.

There are several key features of an ontology that are relevant in the context of egovernment:

- An ontology precisely describes the important concepts in the domain of interest, and the relationships between them.

- The set of important terms and their definitions should be agreed between all participants within the domain, and thus form a basis for communication about the domain.

- The ontology can be specified independently from the specific application for which it is developed, enabling re-use for other e-government purposes.

- An ontology can be formalised and thus support communication between IT systems as well as between humans.

Fundamentally an ontology provides a reference for people to discuss and describe the ways in which they view and interpret a domain. So an ontology can be used to enhance understanding, or as a starting point for people to build models of the domain or a related domain. This was one of our motivations for building the e-Government service ontology, and the ontology forms part of our "framework for e-government services", which will be one of the later deliverables of the project. The framework will be of use to public authorities whether or not they have access to the SmartGov platform.

In general, we would want an ontology to represent the domain as accurately, unambiguously, completely and succinctly as possible. Then, given a particular concept or relationship, we could fully explore its meaning, in terms of associated concepts or relationships. We would, in effect, have a web that described the domain to whatever degree of simplicity or complexity we wanted, given any particular starting point. Such a structure would make an adequate domain map. This was another motivation for building the e-Government service ontology: we could use it as a domain map as shown in Fig. 1

\section{The SmartGov Ontology}

\subsection{Approaches to Ontology Development}

A comprehensive review of ontology development methods appears in [6]. In general, methods are found to:

- take a task as the starting point

- either be stage-based or involve the evolution of prototypes

- involve two separate stages, to derive first an informal description of the domain, then a formal one

- contribute to an anticipated library of connected ontologies

- give some guidance on making choices at a variety of levels 
Ontology developers also almost universally acknowledge the need for iterative refinement of an ontology.

More recently, interest in distributed development of ontologies is reflected in [7], who identified these different approaches to ontology development:

- inspiration: an individual viewpoint about the domain

- induction: description of a specific case within the domain

- deduction: extract general principles about the domain

- synthesis: build the ontology from existing partial characterisations

- collaborate to gain shared viewpoints, using an initial ontology as an anchor

\subsection{Our Approach}

Our approach has been a combination of induction, deduction, synthesis and collaboration. A few points are worth noting.

First, the objective of our ontology was to provide a conceptual framework at the knowledge level, rather than a "concrete artifact at the symbol level, to be used for a given purpose" [8]. This does pose problems in producing a usable domain map in SmartGov: we discuss this later in the paper.

In the sense that the aim of the SmartGov platform is to design transaction services, we did have a task as our starting point, i.e. the task of service design. However, we have tried to take heed of the advice in [6], that task-specific ontology development may limit the reusability of the ontology. Our intention, as stated in the previous section, has been for our e-government services ontology to be usable beyond the SmartGov platform.

We had already built a general view of the government services domain while gathering requirements for the SmartGov platform. We had access to staff at the City of Edinburgh Council (CEC) and in the General Secretariat for Information Systems (GSIS) in the Greek Ministry of Finance.

In addition, we were given access to the results of a wealth of interviews carried out within CEC in the last two years as part of Edinburgh's Smart City initiative to provide services through call centres, one-stop shops and online. From that material we extracted the most frequently-occurring terms and pruned them to a set that we considered to be representative of government services.

We studied the results of several other initiatives to categorise government services, such as $[9,10]$ and extracted terms from them.

We ran a workshop on social acceptance of e-government services, with members of staff at CEC and at Napier University, from which we took further terms that had not appeared elsewhere.

In total we had about 150 terms that described e-government services in general. We avoided being specific about particular services. Examples of the terms are CITIZEN, CONTACT, FORM, LETTER, PAYMENT, BENEFIT, LICENCE, RESPONSIBILITY, TRANSPARENCY, MANDATE and TRUST.

Then began the painstaking task of defining these concepts and relationships in terms of core concepts and of each other. We were greatly assisted in the task by our decision to adopt the Enterprise ontology [11] as a starting point. Its existing 
definitions - particularly in key areas of activity, organisation and market — proved a good basis for many of our definitions, some of which are shown later in the paper.

The Enterprise definitions of MARKET and related terms are based on the notion of SALE. We were somewhat surprised and heartened to find that many of these Enterprise terms translated very easily into the terms in our ontology to do with SERVICE. The Enterprise ontology was also influential in the mode of thinking that we adopted in producing our definitions.

Having created the natural-language definitions, we then formalised our definitions by producing a version of the ontology in the Resource Description Framework (RDF) [12]. This exercise tested the validity of our natural language definitions, several of which had to be revisited and changed. Some additional terms had to be introduced. We used the OIModeler component of the KAON tool set (see Acknowledgements) to generate the RDF.

Finally, feedback was sought from the public authorities and the previous steps were repeated. At the time of writing, this has only been achieved through discussion and workshops. Deployment trials of the SmartGov platform will yield more feedback and lead to further iteration through the ontology development steps.

\subsection{Our Results}

Below are some of the definitions from our ontology. A full list is available in the Results section of the SmartGov project web site (http://www.smartgov-project.org).

In the definitions below, a word in CAPITALS is defined in the e-government services ontology; a word in BOLD CAPITALS was already defined in the Enterprise ontology; a word with an Initial capital is a meta term; a word in italics is a fundamental concept that needs no definition.

The accompanying diagrams, which we produced using the KAON OIModeler, are visual representations of relevant parts of the ontology. We do not describe them in detail here: they are included to give a sense of the complexity of the ontology.

ENQUIRE: ACTIVITY in which a LEGAL ENTITY states their desire for INFORMATION

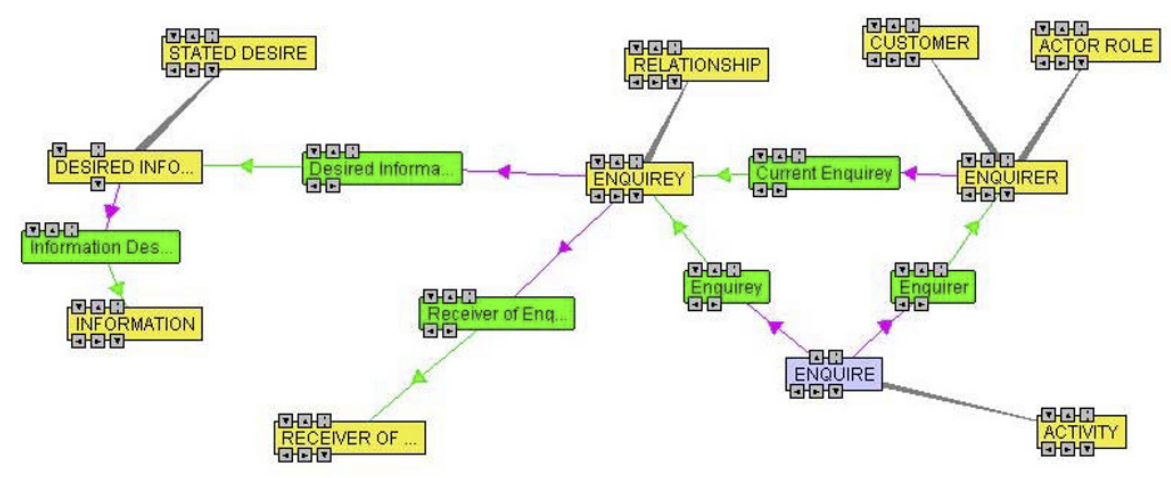


reward: a resource, authority or responsibility given by one legal entity to another on achievement of an activity for which the other legal entity has responsibility

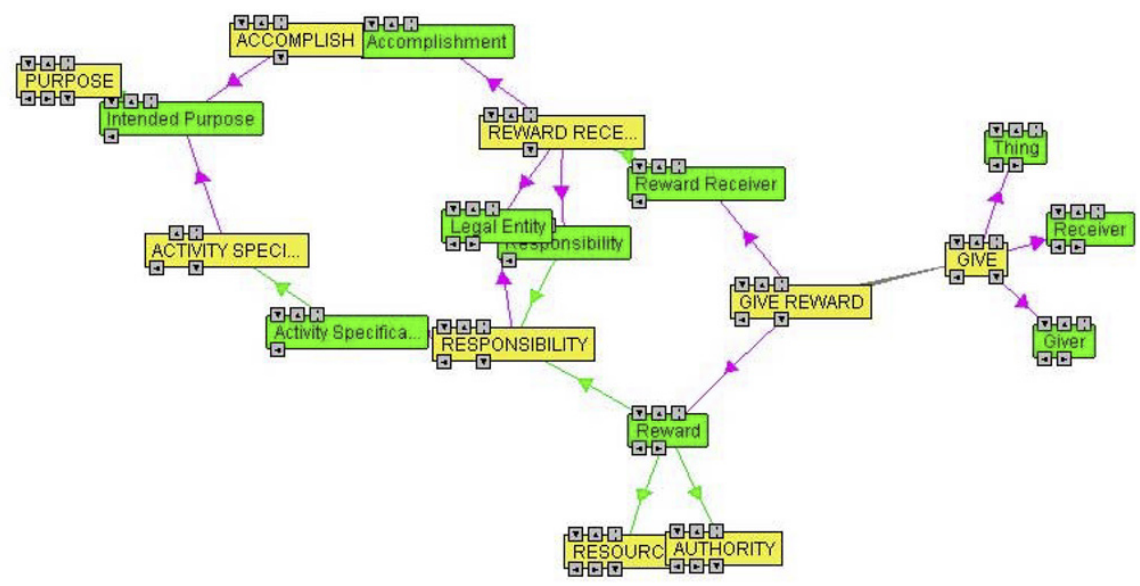

SENSE OF SECURITY: a Relationship between a LEGAL ENTITY and a State of Affairs in which the LEGAL ENTITY has BELIEF that the State of Affairs is SECURITY

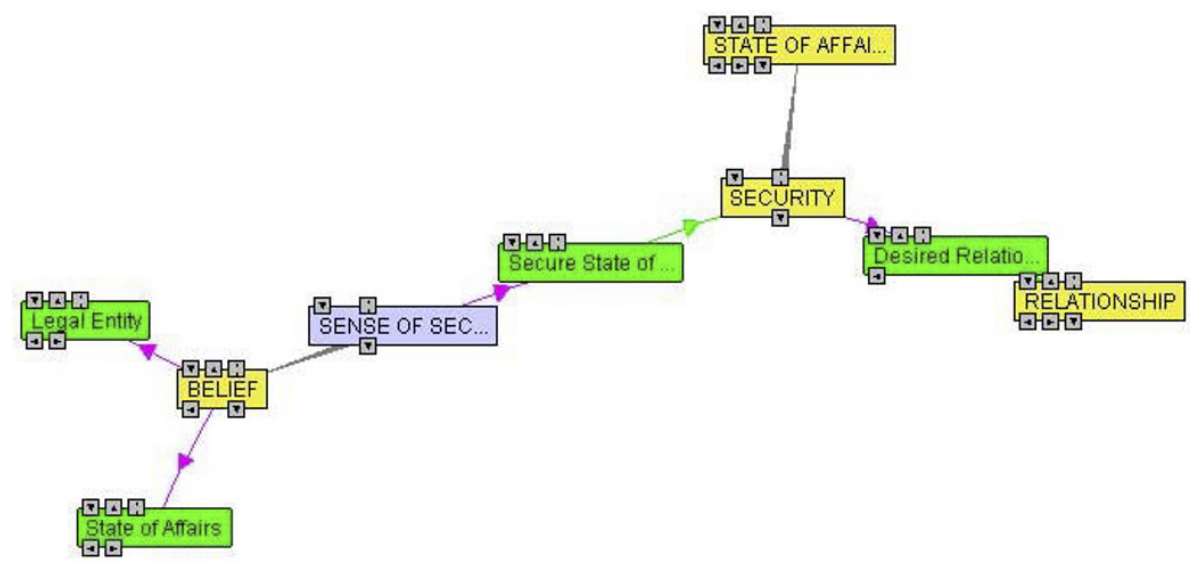

ACCOUNTABILITY: a Relationship between LEGAL ENTITIES in which one must JUSTIFY their ACTIVITIES to the other

APPLICATION: a Relationship between two LEGAL ENTITIES in which one states its desire for a RESOURCE or AUTHORITY from the other

FRONT DESK: a COMMUNICATION MEDIUM between a PUBLIC AUTHORITY and a CUSTOMER in which the Role of the PUBLIC AUTHORITY is played by a PERSON and the GIVING of INFORMATION is IMMEDIATE

NEED: a Relationship between a LEGAL ENTITY and a State of Affairs that is the difference between the true State of Affairs and a defined standard State of Affairs 


\section{The SmartGov Domain Map}

As mentioned above, the ontology provides a conceptual description of e-government services. In general, it can be represented in RDF as a cyclic graph and is a complex, interwoven structure. This poses problems for its use as a domain map, both from the point of view of public authority staff maintaining the ontology, and from the point of view of the knowledge management system navigating the structure.

So, we decided to explore the use of a simplification of the ontology, in the form of a directed acyclic graph.

This graph was extracted directly from the ontology, starting from a set of relevant top-level concepts that adequately describe public authority service provision. These top-level concepts form the parents of searchable taxonomies, made up of concepts and structure taken directly from the ontology.

The top-level concepts are activities, actors, issues, legislation, needs, process, requirements, responsibilities, results, rights and service types.

The subsequent structure has been engineered to enable searchability and function. Each concept has an optimal number of children, which represent the domain as accurately as possible, while at the same time creating a logical search path to give an unambiguous route to the desired target. See Fig. 2.

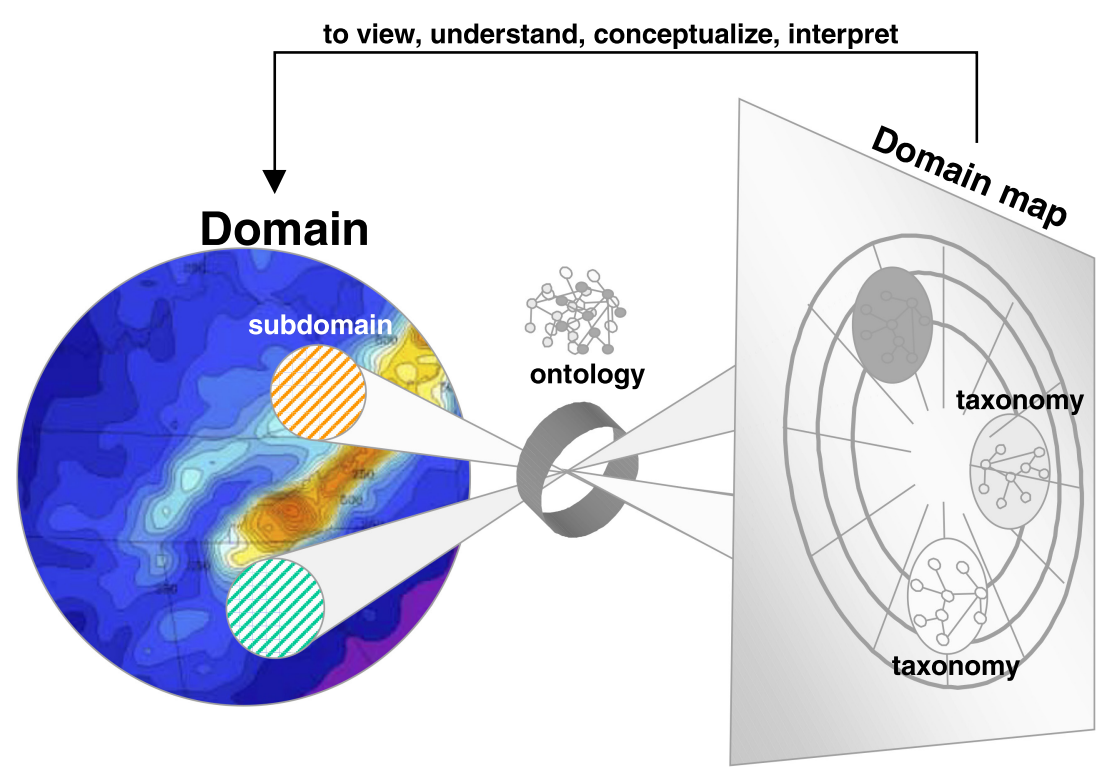

Fig. 2. Taxonomies as a domain map 


\section{Discussion}

Using the Enterprise Ontology as a starting point proved invaluable. We did not need to reinvent the wheel and it set the style of the mode of thinking required to develop our e-government service ontology.

We are in little doubt that the existence of an ontology helps understanding, sharing and the process of building models. An apparent difficulty is the effort required to build and maintain the ontology. The people who matter - public authority staff — have constant operational duties and, even if they can spare the time, are not able to switch quickly into an "ontology" frame of mind.

Technically, too, maintenance poses extra problems. Most current effort in ontology maintenance, e.g. the Onto-logging project [13], is technology-based: how to maintain the integrity of the ontology when new terms are introduced or existing terms are changed. [14] describes the use of a taxonomic domain map (described as an ontology but, to our minds, not meeting the criteria for an ontology) to help people to browse multiple databases. The project has developed new ways of automating the construction and maintenance of their taxonomies.

There is, of course, another dimension: collaboration of people, either with or without the aid of technology. [7] describes a Delphi approach ([15]). We are currently considering whether Delphi-style methods might help us in ontology discussion and maintenance, particularly in view of the time pressure on staff mentioned above.

An additional problem for us is that, within the SmartGov platform, we are using an extraction of the ontology, not the full ontology, as our domain map. This means double maintenance - of the ontology and of the domain map - and the extra burden of keeping them consistent as public authorities want to change one or the other.

We will gather valuable evidence and experience of these issues as SmartGov is deployed, configured and evaluated in our participating public authorities. The project is due to finish in January 2004.

\section{Summary}

The main benefits from the use of the SmartGov e-government ontology are:

- The ontology supports communication by providing a shared vocabulary with welldefined meaning, avoiding ambiguities and misunderstandings. It can support communication between human agents and software agents.

- It is capable of providing flexible support for non-trivial e-transaction services. In the case of SmartGov it allows different aspects of the domain - knowledge units and transaction service components — to be interrelated to best advantage. 
Acknowledgements. SmartGov (IST-2001-35399) is supported by the European Commission under the Information Society Technologies 5th Framework Programme (Key Action Line: On-line support for democratic processes).

We used the software tool OIModeler, which is part of KAON (The Karlsruhe Ontology and Semantic Web Framework), an open-source ontology management infrastructure developed by Forschungszentrum Informatik (FZI) and the Institut für Angewandte Informatik und Formale Beschreibungsverfahren (AIFB), Universität Karlsruhe. KAON is available at http://kaon.semanticweb.org/

Adrianos Evangelidis and Alexander Xenakis contributed valuable comments.

\section{References}

[1] Bertelsmann: Balanced E-Government - Connecting Efficient Administration and Responsive Democracy. Bertelsmann Foundation Publishing House, (2001)

[2] Howard, M.: e-Government Across the Globe: How Will "e" Change Government? Government Finance Review 2001 (2001) 6-9

[3] Layne, K., Lee, J.: Developing fully functional E-government: A four stage model. Government Information Quarterly 18 (2001) 122-136

[4] Papantoniou, A., Hattab, E., Afrati, F., Kayafas, E., Loumos, V.: Change Management, a critical success factor for e-Government. In: Proc. 12th IEEE International Conference and Workshop on Database and Expert Systems Applications DEXA 2001 (2001)

[5] Webera, R., Ahab, D. W., Becerra-Fernandez, I.: Intelligent lessons learned systems. Expert Systems with Applications 17 (2001) 17-34

[6] Jones, D. M., Bench-Capon, T. J. M., Visser, P. R. S.: Methodologies for Ontology Development. In: Proc. IT\&KNOWs (1998)

[7] Holsapple, C. W., Joshi, K. D.: A collaborative approach to ontology design. Communications of the ACM 45 (2002) 42-47

[8] Guarino, N., Giaretta, P.: Ontologies and Knowledge Bases: Towards a Terminological Clarification. ISO Press (1999)

[9] APLAWS. APLAWS Category List.[Online]. Available: http://www.aplaws.org.uk/products/product_acl.cfm

[10] LEAP. LEAP CUPID List. Life Events Access Project [Online]. Available: http://www.leap.gov.uk/xpedio/groups/public/documents/standards/000383.pdf

[11] Uschold, M., King, M., Moralee, S., Zorgios, Y.: The Enterprise Ontology. Knowledge Engineering Review 13 (1998)

[12] Miller, E. (1998) An Introduction to the Resource Description Framework. Corporation for National Research Initiatives [Online]. Available: http://www.dlib.org/dlib/may98/miller/05miller.html

[13] Cañadas, R.: Onto-logging Project Description. Indra, Archetypon, Deltatec, FZI, Insead, Meta4, IST-2000-28293 deliverable D0A (2002)

[14] Hovy, E.: Using an Ontology to Simplify Data Access. Communications of the ACM 46 (2003) 47-49

[15] Turoff, M., Hiltz, S. R.: Computer Based Delphi Processes. In: M. Adler and E. Ziglio, (eds.): Gazing Into the Oracle: The Delphi Method and Its Application to Social Policy and Public Health. Kingsley Publishers (1996) 